A LETTERS JOURNAL EXPLORING THE FRONTIERS OF PHYSICS

\title{
OFFPRINT
}

\section{The Eulerian description of dilute collisionless suspension}

\author{
G. Boffetta, A. Celani, F. De Lillo and S. Musacchio \\ EPL, 78 (2007) 14001
}

Please visit the new website www.epljournal.org 


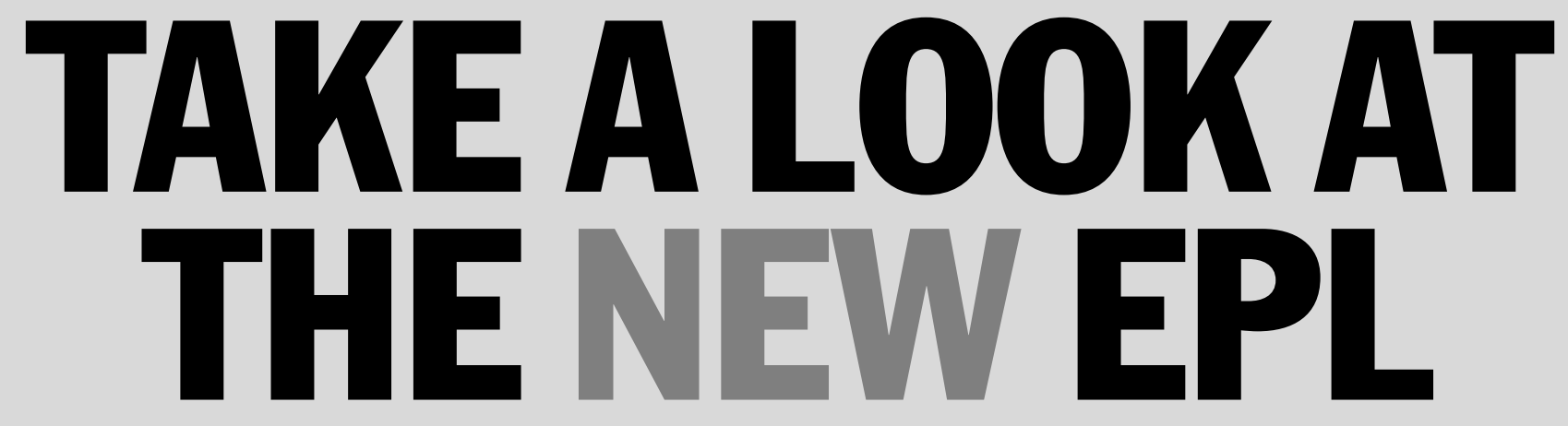

\section{Europhysics Letters (EPL) has a new online home at www.epljournal.org}

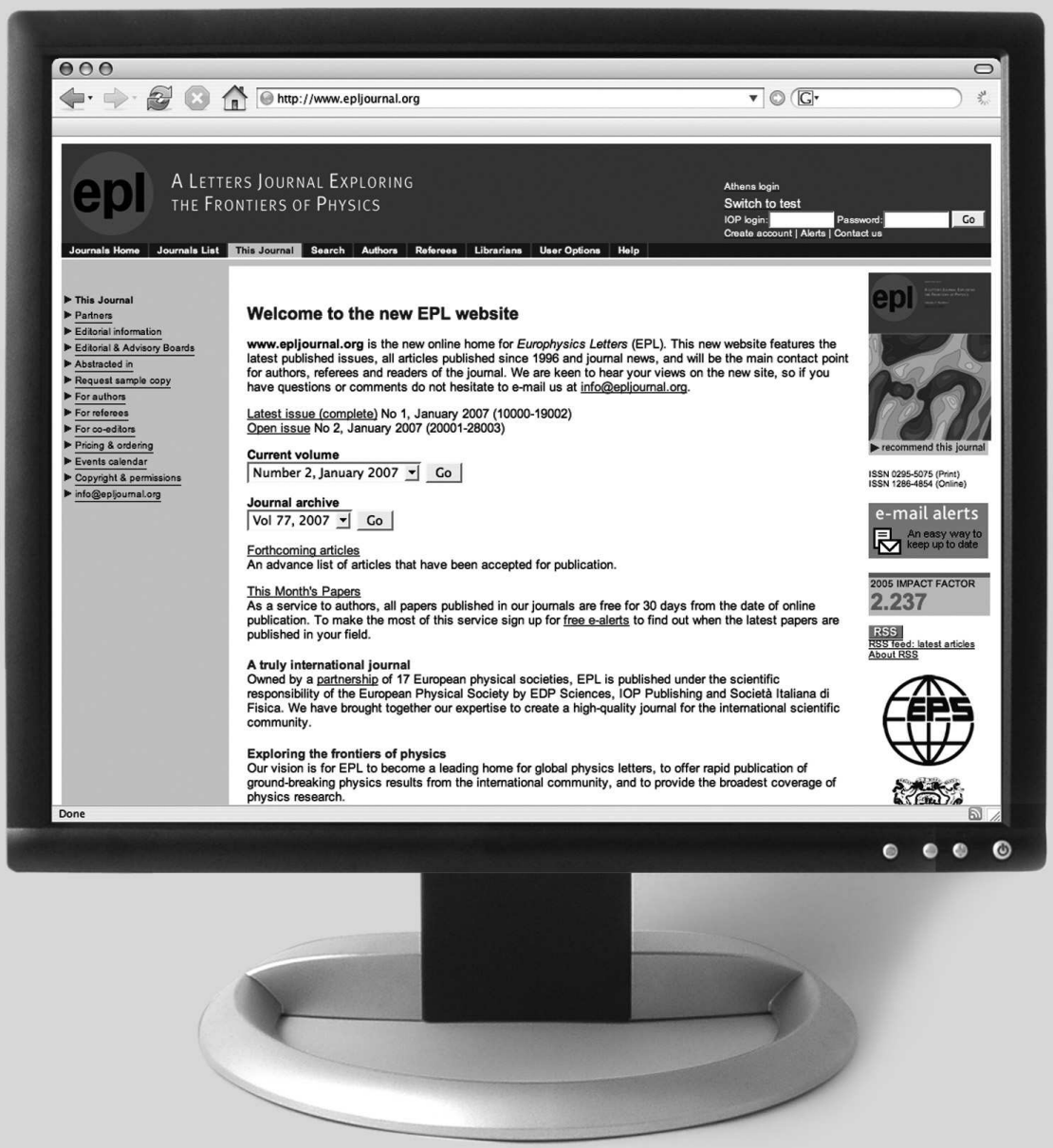

Take a look for the latest journal news and information on:

- reading the latest articles, free!

- receiving free e-mail alerts

- submitting your work to EPL 


\title{
The Eulerian description of dilute collisionless suspension
}

\author{
G. Boffetta ${ }^{1}$, A. Celani ${ }^{2}$, F. De Lillo $^{3}$ and S. Musacchio ${ }^{2}$ \\ ${ }^{1}$ Dipartimento di Fisica Generale and INFN - Via Pietro Giuria, 1, 10125, Torino, Italy and \\ CNR-ISAC, Sezione di Torino - C. Fiume 4, 10133, Torino, Italy \\ ${ }^{2}$ INLN-CNRS - 1361 Route des Lucioles, Sophia Antipolis, 06560 Valbonne, France \\ ${ }^{3}$ Fachbereich Physik, Philipps-Universität - Renthof 6, 35032 Marburg, Germany
}

received 11 October 2006; accepted in final form 15 February 2007

published online 15 March 2007

PACS 47.27.Gs - Isotropic turbulence; homogeneous turbulence

\begin{abstract}
We analyze the statistical properties of a Eulerian fluid model describing the evolution of a suspension of inertial particles in an incompressible flow. Regularity and compressibility of the velocity field for the inertial phase are investigated in the limit of heavy particles by means of numerical simulations in two- and three-dimensional flows. We show that in the small Stokes number regime the Eulerian fluid model is able to capture fine details of the clustering dynamics, and exhibits good agreement with fully Lagrangian simulations of inertial particle trajectories. The fluid description breaks down due to collisions at Stokes numbers $\gtrsim 0.1$, the actual value depending on the carrier flow characteristics.
\end{abstract}

Copyright (c) EPLA, 2007

The advection of small heavy impurities suspended in incompressible flows is relevant for several engineering and environmental applications, ranging from Diesel engines [1] to the formation of rain droplets in warm clouds $[2,3]$, to the dispersion of dust in the environment [4]. From a theoretical point of view, inertial particle dynamics is of general interest because of the spontaneous clusterization that takes place even in a perfect incompressible flow (the so-called preferential concentration effect [5]). In the last years there has been considerable progress in our understanding of inertial particle dynamics, mainly on the basis of numerical $[6,7]$ and theoretical $[3,8-10]$ results.

A natural description of inertial suspensions in turbulent flows can be given in a mixed Eulerian-Lagrangian framework. The fluid phase is described in terms of a Eulerian field (the velocity field) while the particles, assumed to be very dilute, are described in terms of individual Lagrangian trajectories. A difficulty of this approach is the lack of a rigorous way to incorporate the particle feedback to the fluid governing equation in the case of active particles, i.e. when the effects of the inertial phase on the fluid phase cannot be neglected.

To address this issue, in the recent past several Eulerian multiphasic approaches have been proposed [10-16]. The particulate phase is here treated as a continuum, as well as the carrier phase. This description provides a natural framework for the theoretical and numerical study of the feedback. As a counterpart this approach requires the numerical solution of the additional equation for the particulate phase, and hence is often computationally more demanding than the Lagrangian one. Nevertheless, a Eulerian description turns out to be convenient for a detailed study of particle clustering, which requires a huge number of particles in the Lagrangian approach to resolve regions of low concentration.

In the present letter, we investigate the statistical properties of a Eulerian model for suspensions of monodisperse inertial particles. We consider very dilute suspensions, where both particle-to-particle interactions (e.g. collisions) and feedback of the particles on the fluid are neglected (passive limit). The dynamics of the fluid phase is hence independent of the particle motion, and is given by an additional equation of motion (e.g., Navier-Stokes). The solid phase can be described as an ensemble of particles, characterized by the probability density function (pdf) $P(\boldsymbol{x}, \boldsymbol{q}, t)$ to find a particle at time $t$ at position $\boldsymbol{x}$ with velocity $\boldsymbol{q}$.

A widely accepted model for describing the dynamics of a single spherical inertial particle suspended in a fluid has been proposed by Maxey and Riley in the limit of small particle-based Reynolds number [17]. Two parameters control the particle motions: the added-mass factor $\beta=$ $3 \rho_{f} /\left(\rho_{f}+2 \rho_{p}\right)$, which discriminates between heavy $(\beta<1)$ and light $(\beta>1)$ particles, and the Stokes response time $\tau_{s}=a^{2} /(3 \beta \nu)$. Here $a$ is the particle radius, $\rho_{p}$ and $\rho_{f}$ are 
the particle and fluid density, respectively, and $\nu$ is the kinematic viscosity of the fluid.

Neglecting the effects of gravity and the Faxen correction as well as the Basset-Boussinesq history force, the Lagrangian equations for particle trajectory $\boldsymbol{X}(t)$ and velocity $\boldsymbol{V}(t)=\dot{\boldsymbol{X}}(t)$ reduce to $[17]$

$$
\left\{\begin{aligned}
\frac{\mathrm{d} \boldsymbol{X}}{\mathrm{d} t} & =\boldsymbol{W}(t)+\beta \boldsymbol{u}(\boldsymbol{X}(t), t), \\
\frac{\mathrm{d} \boldsymbol{W}}{\mathrm{d} t} & =\frac{(1-\beta) \boldsymbol{u}(\boldsymbol{X}(t), t)-\boldsymbol{W}(t)}{\tau_{s}},
\end{aligned}\right.
$$

where $\boldsymbol{u}(\boldsymbol{x}, t)$ is the incompressible fluid velocity field, and $\boldsymbol{W}(t)=\boldsymbol{V}(t)-\beta \boldsymbol{u}(\boldsymbol{X}(t), t)$ is the covelocity of the particle.

The Fokker-Planck equation for the pdf of the inertial phase of a dilute suspension, $P(\boldsymbol{x}, \mathbf{v}, t)$, is obtained from (1) in a straightforward way when particle-to-particle interactions are neglected (see [10] for a general discussion of this procedure). In the following, we will consider the simplest Eulerian model which is obtained by further assuming that particle velocity is uniquely defined in every point in space by a smooth field $\boldsymbol{v}(\boldsymbol{x}, t)$, different from the fluid velocity $\boldsymbol{u}(\boldsymbol{x}, t)$. This is tantamount to factorize the pdf of particles as $P(\boldsymbol{x}, \boldsymbol{q}, t)=n(\boldsymbol{x}, t) \delta(\boldsymbol{q}-\boldsymbol{v}(\boldsymbol{x}, t))$, where the field $n(\boldsymbol{x}, t)=\int P(\boldsymbol{x}, \boldsymbol{q}, t) \mathrm{d} \boldsymbol{q}$ represents the local number density of particles. The resulting equations for the density and covelocity fields $\mathbf{w}=\boldsymbol{v}-\beta \boldsymbol{u}$ are:

$$
\left\{\begin{array}{l}
\partial_{t} n+\nabla \cdot((\boldsymbol{w}+\beta \boldsymbol{u}) n)=0, \\
\partial_{t} \boldsymbol{w}+(\boldsymbol{w}+\beta \boldsymbol{u}) \cdot \nabla \boldsymbol{w}=\frac{(1-\beta) \boldsymbol{u}-\boldsymbol{w}}{\tau_{s}} .
\end{array}\right.
$$

Let us remark that, for generic flows, the existence of the velocity field $\boldsymbol{v}(\boldsymbol{x}, t)$ is not guaranteed at all times. Indeed, due to their inertia, particles can detach from the fluid trajectories, and can simultaneously cross each other with non-zero relative velocity (the so-called sling effect $[3,18])$. This corresponds to the formation of a shock in the particle velocity field, which becomes ill-defined, and of caustics in the particle density [19]. This phenomenon is driven by strong gradients of the fluid velocity field $\boldsymbol{u}$, while it is contrasted by the linear relaxation to $\boldsymbol{u}$, which reduces the probability of formation of shocks. The relative strength of these competing effects is measured by the nondimensional Stokes number $S t=\lambda \tau_{s}$, where $\lambda$ is the (Lagrangian) Lyapunov exponent of the fluid flow [20]. We remark that an alternative definition of Stokes number $S t=\tau_{s} / \tau_{\eta}$ based on Kolmogorov time $\tau_{\eta}$ is often used. For a turbulent flow the two quantities are related by the empirical formula $\lambda \tau_{\eta} \simeq 0.1$ (see e.g., [21]).

In the simplified situation of random incompressible flows, it has been shown that the probability of observing the formation of shocks decreases exponentially in the limit of vanishing inertia $[19,22]$, thus one can expect that the fluid model (2) reproduces with good accuracy the Lagrangian dynamics of inertial particles in this limit.

For a numerical study of the fluid model, in the following we specialize to the limit of heavy particles $\rho_{p} \gg \rho_{f}$, for which $\beta \rightarrow 0$ and the covelocity reduces to the particle velocity. In this limit, the velocity equation in (2) simplifies to

$$
\frac{\partial \boldsymbol{v}}{\partial t}+\boldsymbol{v} \cdot \nabla \boldsymbol{v}=\frac{\boldsymbol{u}-\boldsymbol{v}}{\tau_{s}}
$$

formally an inviscid Burgers equation with linear relaxation to the fluid velocity $\boldsymbol{u}$. In the absence of a pressure term, which would preserve incompressibility, the solution of (3) will in general present a compressible component (i.e. $\nabla \cdot \boldsymbol{v} \neq 0$ ). The fluid phase is governed by the incompressible Navier-Stokes equation

$$
\frac{\partial \boldsymbol{u}}{\partial t}+\boldsymbol{u} \cdot \nabla \boldsymbol{u}=-\nabla p+\nu \Delta \boldsymbol{u}+\boldsymbol{f}
$$

with $\nabla \cdot \boldsymbol{u}=0$.

Numerical simulations are performed by integrating eqs. (3) and (4) by means of a fully dealiased pseudospectral code in two and three dimensions at resolution $N^{2}=$ $256^{2}$ and $N^{3}=64^{3}$ with 2nd-order Runge-Kutta timestepping. In order to avoid complications induced by the presence of material boundaries, we consider the case of periodic boundary conditions. The flow is sustained by a large-scale random forcing $f$. Kinematic viscosity $\nu$ is chosen large enough to resolve in detail the viscous dissipative regime, where clustering of inertial particles takes place.

As a first test of the Eulerian model we consider the spatial distribution of particles which move according to the inertial velocity field $\boldsymbol{v}$ given by eq. (3),

$$
\frac{\mathrm{d} \tilde{\boldsymbol{X}}}{\mathrm{d} t}=\boldsymbol{v}(\tilde{\boldsymbol{X}}, t)
$$

and compare it with the distribution of inertial particles obtained by integration of the Lagrangian model (1). The two sets of particles are seeded randomly in the same two-dimensional turbulent flow $\boldsymbol{u}$, with identical spatial distributions at time $t=0$. We remark that the two dynamical systems (1) and (5) are formally very different as the dimensionality of the former is double (the phase space includes both position and velocity). Despite this important difference, we find that the behavior of particles in the two models is very similar for moderate values of $S t$.

Figure 1 shows two snapshots of inertial particle distribution obtained by integrating the two models at $S t=$ 0.063. The qualitative agreement is remarkable. In both cases we observe a highly inhomogeneous distribution with large empty regions and strong concentration on filamental structure. The variance of particle density $\left\langle n^{2}(\boldsymbol{x}, t)\right\rangle-\langle n(\boldsymbol{x}, t)\rangle^{2}$, shown in fig. 2 (left panel), confirms that at small value of Stokes number the fluid model recovers with considerable accuracy the fluctuations of particle distribution.

These distributions are a consequence of the dissipative nature of the equation of motion. In the Lagrangian 

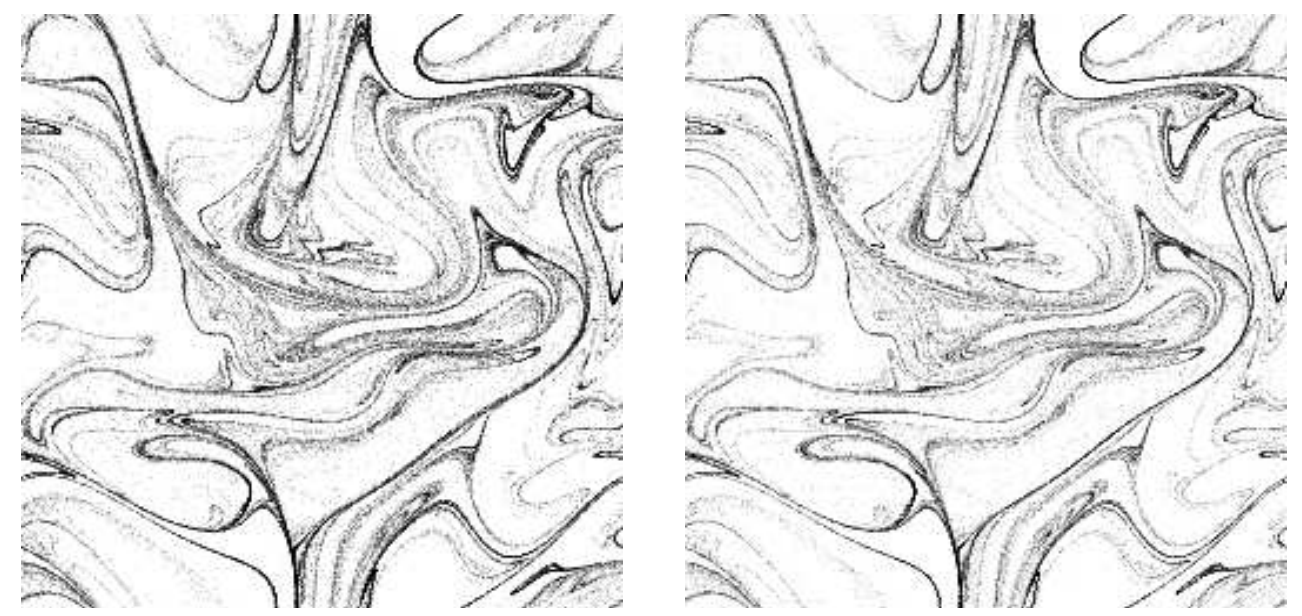

Fig. 1: Snapshots of the inertial particle concentrations obtained from the Lagrangian model (1) with $\beta=0$ (left panel) and the fluid model (3), (5) (right panel). Particles are suspended in a two-dimensional turbulent flow. Stokes number is $S t=0.063$.
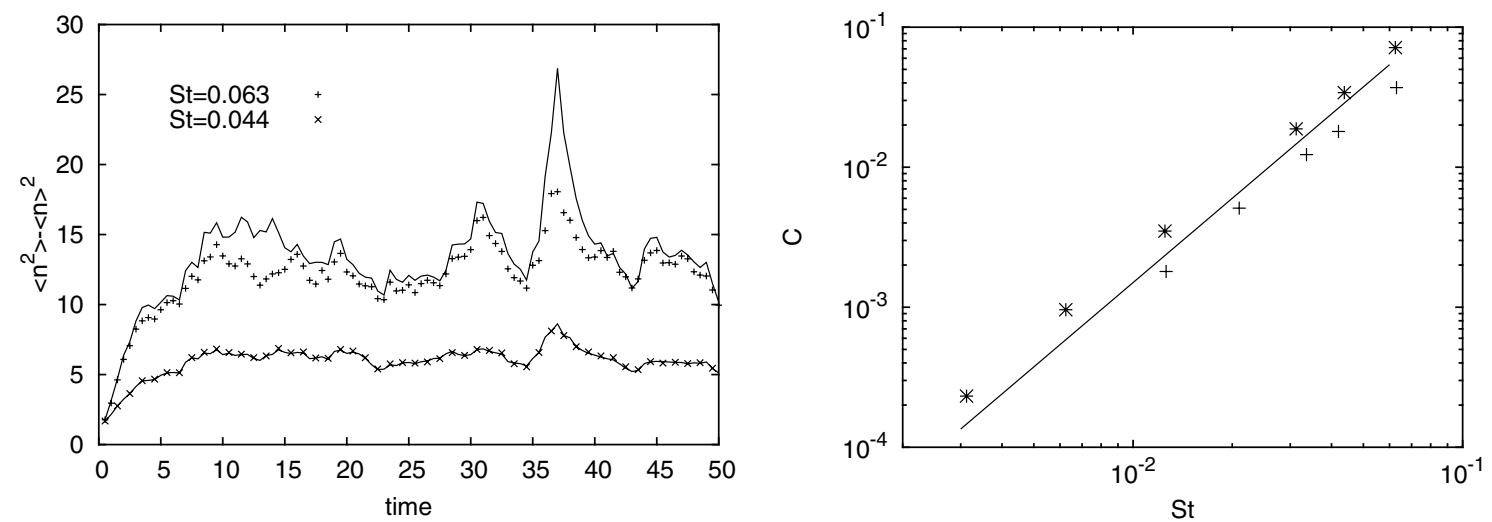

Fig. 2: Left panel: variance of particle density obtained from the fluid model (symbols) and from Lagrangian simulations (lines) in the same 2D turbulent carrier flow. Particles are initially distributed randomly with uniform probability. Right panel: compressibility $\mathcal{C}$ vs. $S t=\lambda \tau_{s}$ of the particle velocity field carried by Navier-Stokes fluid in $2 \mathrm{D}(*)$ and $3 \mathrm{D}(+)$. The line represents the $S t^{2}$ behavior at small $S t$.

model, the contraction rate of phase space is $-d / \tau_{s}$ (in $d$-dimensions) and the long-time behavior on inertial tracers takes place on a multifractal set [9]. In the fluid model inhomogeneity is originated by the compressible nature of the velocity $\boldsymbol{v}$. This effect can be quantified by a standard measure of compressibility given by the dimensionless ratio $\mathcal{C}=\left\langle\left(\partial_{i} v_{i}\right)^{2}\right\rangle /\left\langle\left(\partial_{i} v_{j}\right)^{2}\right\rangle$ which takes values between 0 (incompressible flow) and 1 (potential flow). For small values of $S t$, by expanding (3) at first order in $\tau_{s}$ one has $\boldsymbol{v} \simeq \boldsymbol{u}-\tau_{s}\left(\partial_{t} \boldsymbol{u}+\boldsymbol{u} \cdot \boldsymbol{\nabla} \boldsymbol{u}\right)$ from which $\boldsymbol{\nabla} \cdot \boldsymbol{v} \simeq \tau_{s} \boldsymbol{\nabla}$. $(\boldsymbol{u} \cdot \nabla \boldsymbol{u})$ and thus one expects for small $S t$ the behavior $\mathcal{C} \propto S t^{2}$. Figure 2 shows the average value of compressibility for 2D and 3D turbulent flows in stationary conditions as a function of $S t$. The $S t^{2}$ behavior is clearly observable. Detailed investigations of the first-order expansion, also termed equilibrium Eulerian approach, have been performed by Balachandar and coworkers $[15,16]$. They showed that for small Stokes numbers it faithfully reproduces the statistics obtained from exact Lagrangian simulations. As shown in fig. 2 the average compressibility of the inertial velocity field $\boldsymbol{v}$ obtained from the dynamical model (3) is pretty well captured by its first-order approximation. The complete solution of eq. (3) clearly extends the Stokes-number range of validity of the Eulerian model, and it is more accurate in recovering the details of the spatial distribution of particle concentration, at the cost of solving an additional equation of the same complexity of the one for the fluid phase.

A quantitative measure of particle clustering observed in fig. 1 can be given in terms of the fractal dimension of the distribution. A convenient indicator is the Lyapunov dimension [23] which is obtained from the spectrum of Lyapunov exponents of the particle motion as $d_{L}=J-$ $\sum_{j=1}^{J} \lambda_{j} / \lambda_{J+1}$, where $\lambda_{j}$ are the Lyapunov exponent in non-increasing order and $J$ is the largest integer for which $\sum_{j \leqslant J} \lambda_{j} \geqslant 0$. The Lyapunov dimension for the (2ddimensional) dynamical system (1) has been used for characterizing the fractal distribution of inertial particles 

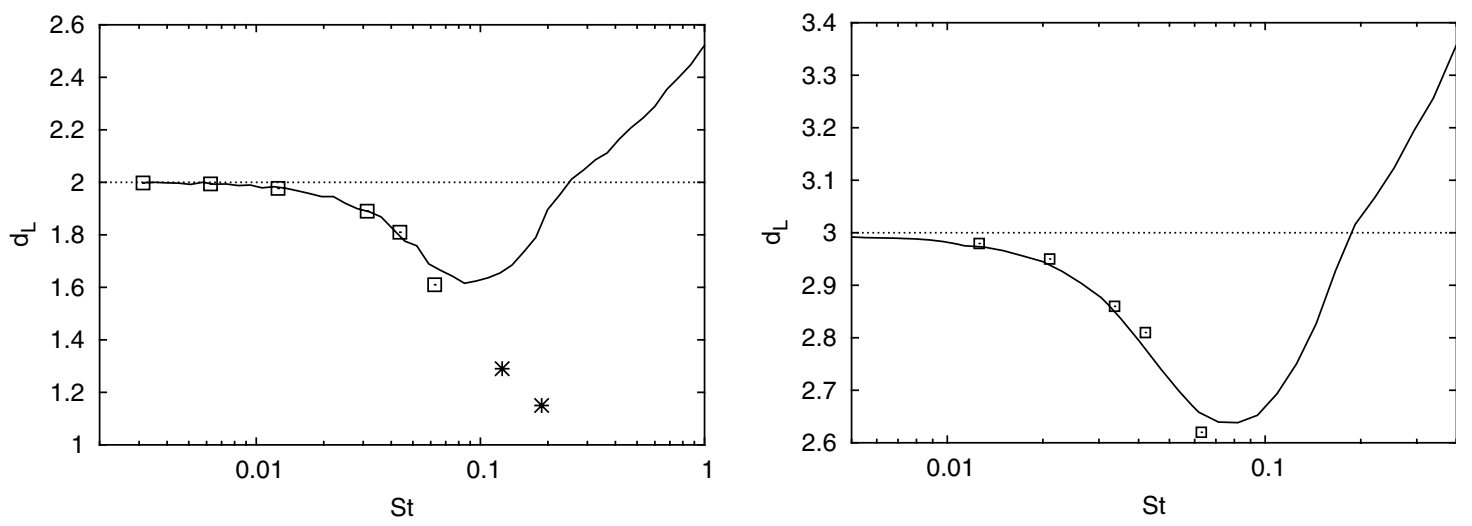

Fig. 3: Left panel: Lyapunov dimension of the attractor for inertial particles following Lagrangian dynamics (1) (line) and fluid model (3), (5) (symbols). A small dissipative term $\kappa \Delta v$ with $\kappa=5 \cdot 10^{-3}$ is added to (3) for $S t>0.07$ (asterisks). Right panel: the same for 3D turbulent flow.
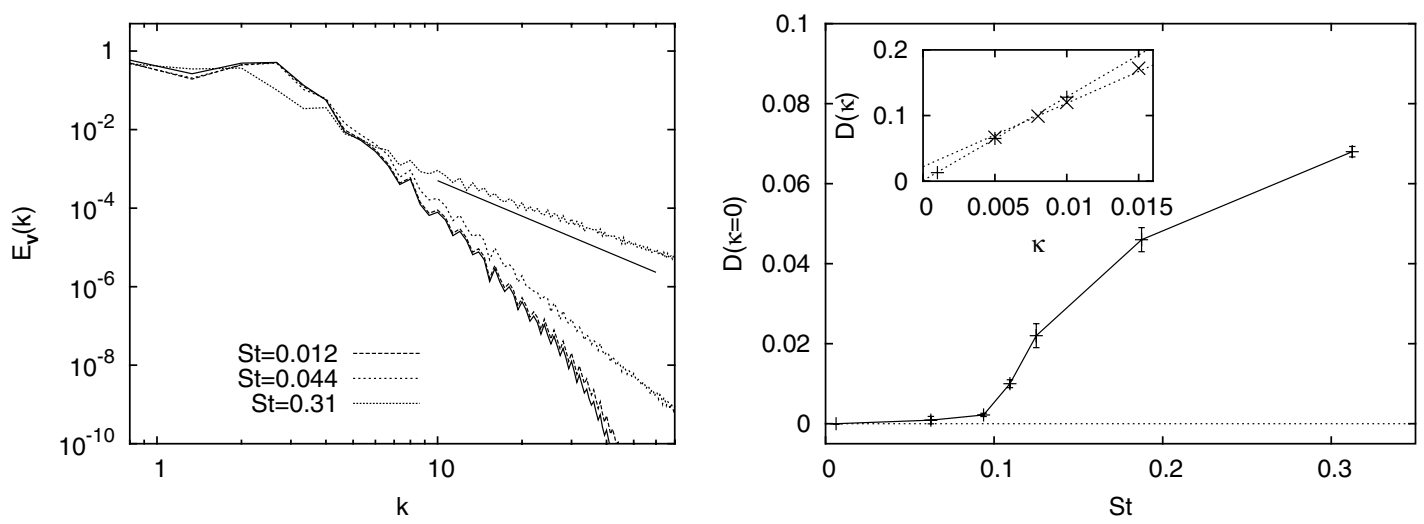

Fig. 4: Left panel: energy spectra of particle velocity field (in the 2D case) for different values of Stokes number (from bottom to top: $S t=0.012, S t=0.044$ and $S t=0.31$ ). We also show the energy spectrum of the carrier fluid (bottom, bold line). At small Stokes numbers the spectra are steeper than the smooth limit $k^{-3}$, represented by the straight line. At $S t=0.31$ the spectrum is less steep than $k^{-3}$, and the simulation is regularized by the dissipative term $(\kappa>0)$. Right panel: dependence of the particle energy dissipation in the limit $\kappa \rightarrow 0$ on the Stokes number, for the case of $2 \mathrm{D}$ direct cascade flow. Values are obtained by a linear extrapolation to $\kappa=0$ of finite dissipation runs, as shown in the inset for $S t=0.063(+)$ and $S t=0.125(\times)$.

in smooth [9] and turbulent [24] flows. The observed asymptotic behavior in this case are $d_{L}=d$ for $\tau_{s} \rightarrow 0$ (fluid particle limit) and $d_{L}=2 d$ for $\tau_{s} \rightarrow \infty[9]$.

Figure 3 shows the result of the Lyapunov dimension as a function of $S t$ for both the original Lagrangian model (1) and the evolution law (5) with inertial velocity given by (3). We remark that the actual behavior of the Lyapunov dimension measured from the Lagrangian trajectories in the $3 \mathrm{D}$ case is in strong agreement with the theoretical results obtained in ref. [8] in the framework of short-correlated flows. The predicted minimum Lyapunov dimension $d_{L} \simeq 2.65$ is confirmed by our findings. The increase of the dimensionality at large Stokes number is due to folding in the phase space of particle attractor. The consequence of such folding is that particle velocity becomes multivalued at a given position. Of course this effect cannot be observed in the $d$-dimensional fluid model in which $\boldsymbol{v}(\boldsymbol{x}, t)$ is single-valued by definition. Therefore the fractal dimension of particle distribution obtained from the fluid model monotonically decreases at increasing the Stokes number, and the minimum of $d_{L}$ cannot be recovered. Notice that for large Stokes numbers the Eulerian approach overpredicts the degree of clustering, as already observed in [15]. Nevertheless, the quantitative agreement between the Lagrangian and fluid model for small $S t \lesssim 0.1$ is remarkable both in $2 \mathrm{D}$ and $3 \mathrm{D}$ turbulent flows.

We remind that the fluid model here discussed relies on the assumption that the inertial velocity can be described by a single-valued field $\boldsymbol{v}$. It is thus necessary that the relaxation of the inertial velocity $\boldsymbol{v}$ toward the fluid velocity $\boldsymbol{u}$ is fast enough to prevent the formation of shocks. In turbulent flows it is not possible to define a priori a critical Stokes number below which the absence 
The Eulerian description of dilute collisionless suspension

of shocks is guaranteed. Still it is possible that in the small Stokes number regime, the statistical weight of eventual shocks is weak enough to allow to neglect them [22].

Shocks can form in a finite time in a velocity field if it is Hölder continuous of order $h$, with $0<h<1$. This corresponds to a power spectrum $E_{v} \equiv(1 / 2) \int|\boldsymbol{v}(\boldsymbol{x})|^{2} \mathrm{~d} \boldsymbol{x}$ with a slope less steep than $k^{-3}$. Conversely, in a smooth velocity field, whose spectral slope is steeper than -3 , an infinite time would be necessary to form a shock. In fig. 4 (left panel) we show the power spectra of particle velocity field for different value of $S t$. We also show, for comparison, the energy spectrum of the twodimensional fluid velocity field $\boldsymbol{u}$. For small values of Stokes number the particle velocity field remains smooth, and the corresponding spectra are steeper than $k^{-3}$, thus preventing the formation of shocks. At $S t \simeq 0.07$ the slope of the spectrum becomes lower than -3 , signaling the presence of sharp fronts in the particle velocity field. In this case a small dissipative term $\kappa \Delta v$ is added to eq. (3) to regularize the velocity fields at small scales.

The behavior of the "particle energy dissipation" $D\left(\tau_{s}\right) \equiv \kappa\left\langle\left(\partial_{i} v_{j}\right)^{2}\right\rangle$ in the limit $\kappa \rightarrow 0$ can be used to detect the formation of shocks in the particle velocity field. The limit $\kappa \rightarrow 0$ is obtained by linear extrapolation of the values of $D\left(\tau_{s}\right)$ measured in a sets of simulations with decreasing values of $\kappa$ (see inset of fig. 4, right panel). For small Stokes number, the dissipation $D\left(\tau_{s}\right)$ vanishes in the limit $\kappa \rightarrow 0$ (see fig. 4 right panel), which indicates the absence of significant singularity in the particle velocity field. This confirms a posteriori the validity of the fluid model in the small Stokes number regime.

In conclusion, we have introduced and numerically investigated a Eulerian fluid model describing the dynamical evolution of a suspension of heavy particles in twodimensional and three-dimensional incompressible flows. We have shown that the Eulerian model is able to reproduce with high accuracy the detailed structure of particle concentration as obtained from the reference Lagrangian model up to moderate Stokes numbers. For larger $S t>0.1$, where the sling effect becomes important, the Eulerian model is unable to reproduce the observed reduction of particle clustering. This failure can be interpreted as a consequence of a substantial increase in the probability of shock formation in the particle velocity field. This is numerically confirmed by investigation of the spectra of the inertial particle velocity field.

The crucial limitation of the Eulerian model is that it does not allow to deal with the folding in phase space associated to the formation of caustics. Its range of validity is hence restricted to small Stokes numbers. In this regime, where the statistical role of singularities is negligible, it provides an effective tool for the investigation of inertial particle dynamics. In particular it offers a suitable framework for theoretical and numerical study of the feedback of the particulate phase. The extension of fluid models to the collision regime, along the lines proposed in ref. [18] and exploiting recent results on the collision rate presented in ref. [25], is one of the most interesting challenges for the near future.

$$
* * *
$$

The authors thank E. Meiburg for pointing out the works by Balachandar et al. $[15,16]$. Part of the simulations were done by FD while at INLN, supported by the European network HPRN-CT-2002-00300.

\section{REFERENCES}

[1] Post S. and Aвraham J., Int. J. Multiphase Flow, 28 (2002) 997.

[2] Pinsky M. and Khain A., J. Aerosol Science, 28 (1997) 1177.

[3] Falkovich G., Fouxon A. and Stepanov M. G., Nature (London), 419 (2002) 151.

[4] Seinfeld J., Atmospheric Chemistry and Physics of Air Pollution (J. Wiley and Sons, New York) 1986.

[5] Squires K. D. and Eaton J. K., Phys. Fluids, 3 (1991) 1169.

[6] Reade W. C. and Collins L. R., J. Fluid Mech., 415 (2000) 45.

[7] Chun J., Koch D. L., Rani S., Ahluwalia A. and Coluins L. R., J. Fluid Mech., 536 (2005) 219.

[8] Duncan K., Mehlig B., Östlund S. and Wilkinson M., Phys. Rev. Lett., 95 (2005) 240602.

[9] BeC J., Phys. Fluids, 15 (2003) L81.

[10] Jackson R., Chem. Eng. Sci., 52 (1997) 2457.

[11] Zhang D. R. and Prosperetti A., Phys. Fluids, 6 (1994) 2956.

[12] Zhang D. R. and Prosperetti A., Int. J. Multiphase Flow, 23 (1997) 425.

[13] Druzhinin O. A. and Elghobashi S., Phys. Fluids, 10 (1998) 685.

[14] Druzhinin O. A. and Elghobashi S., Phys. Fluids, 11 (1999) 602.

[15] Rani S. L. and Balachandar S., Int. J. Multiphase Flow, 29 (2003) 1793.

[16] Shotorban B. and Balachandar S., Phys. Fluids, 18 (2006) 065105.

[17] Maxey M. R. and Riley J. J., Phys Fluids, 26 (1983) 883.

[18] Falkovich G. and Pumir A., preprint nlin.CD/0605040 (2006).

[19] Wilkinson M. and Mehlig B., Europhys. Lett., 71 (2005) 186.

[20] Crisanti A., Falcioni M., Paladin G. and Vulpiani A., Riv. Nuovo Cimento, 14, No. 12 (1991) 1.

[21] Bec J., Biferale L., Boffetta G., Cencini M., Musacchio S. and Toschi F., Phys. Fluids, 18 (2006) 091702.

[22] Derevyanko S., Falkovich G., Turitsyn K. and TURITSYN S., preprint nlin.CD/0602006 (2006).

[23] Отт E., Chaos in Dynamical Systems (Cambridge University Press, New York) 1993.

[24] Boffetta G., De Lillo F. and Gamba A., Phys. Fluids, 16 (2004) L20.

[25] Wilkinson M., Mehlig B. and Bezuglyy V., Phys. Rev. Lett., 97 (2006) 048501. 